\title{
Functional Tests Assessing Return to Activity after Anterior Cruciate Ligament Reconstruction
}

\author{
Eun Wook Chang \\ Department of Kinesiology, Inha University, Incheon, Korea
}

PURPOSE: A successful return to activity following anterior cruciate ligament reconstruction is the primary goal for athletes and physically active populations who underwent the surgery. However, functional tests that could be utilized for deciding return to activity are not well-described. Therefore, we reviewed currently suggesting functional tests and the effectiveness of each tests.

METHODS: In this review, we summarized the patient oriented subjective functional measures, objective functional tests, a functional test battery, and a general pass and fail criteria for functional tests.

RESULTS: There are various subjective and objective functional measures to assess ACLR patients' level of knee function. The concept of a functional test battery that is the combination of various functional tests is more sensitive to detect patient's readiness to return. Eighty-five to ninety percent of limb symmetry index score is frequently applied as the pass and fail criteria for functional test.

CONCLUSIONS: Various researches suggested functional tests that clinicians could be applied to their patients for return to activity safely. Despite the use of various functional tests, the risk for subsequent knee problems remains elevated in ACLR individuals. Therefore, it is necessary to investigate the usefulness of functional tests to identify individuals that are at greatest risk for subsequent knee injury.

Key words: Anterior cruciate ligament injury, Functional test, Return to activity, Functional test battery

\section{INTRODUCTION}

Clinicians, scientists and other rehabilitation professionals continue to try to optimize the anterior cruciate ligament (ACL) rehabilitation process and develop return to full activity criteria for anterior cruciate ligament reconstructed patients that identify readiness of patients' knee function to minimize second injury risks. However, despite the best efforts of rehabilitation clinicians, patients that are able to return to full activity following anterior cruciate ligament reconstruction (ACLR) are at significantly greater risk of sustaining a second ACL injury event [1,2]. There are number of different functional measurements that are currently used to determine a patient's level of function and readiness to return to activity. Patient-oriented subjective tests that can be used to evaluate a patient's knee function include the International Knee Documentation Committee 2000 Subjective Knee Form (IKDC 2000), Knee Out- come Survey Activities of Daily Living Scale (KOS-ADLS), and Global Rating Scale of Perceived Function (GRS). Examples of objective tests that can be used in clinical practice include time since post-ACLR, muscle strength, thigh circumference, knee joint range of motion, and singleleg hop tests. There are two purpose of this review. The first purpose is to introduce and discuss about various subjective and objective measures, methods to decide cut-off standards, and the use of a functional test battery (FTB) are introduced and discussed. The second purpose is to provide practical information for clinicians to properly implement the functional test in the practice.

\section{PATIENT-ORIENTED SUBJECTIVE MEASURES OF FUNCTION}

The IKDC 2000 subjective knee evaluation consists of three sections 
with a total of 19 items-7 questions on symptoms, 10 questions on sports activities, and 2 questions related to function. Each patient responds to questions with individual scores using a Likert scale that indicates their current level of activity. The IKDC 2000 is scored by summing the individual question scores and then transforming the total score to a scale from 0 to 100 using the following equation:

$$
\text { IKDC Score }=\left(\frac{\text { Sum of Items Answered }}{\text { Maximum Possible Score }}\right) \times 100
$$

Previous studies have reported high reliability and construct validity when using the IKDC to evaluate the level of knee function. Test-retest reliability of the IKDC has been reported between 0.90 and 0.95 for patients with knee injuries including ACL, meniscus, and chondral defects $[3,4]$. The IKDC score is highly correlated with other patient-oriented subjective tests such as the Cincinnati Knee Rating System, pain visual analog scale, Lysholm score and SF-36 physical component and physical function $[5,6]$. However, IKDC 2000 showed a lack of content validity because of no patients' contribution in developing this questionnaire. The normative database of the IKDC 2000 can be used as a standard of successful patient-reported outcome [7] and the IKDC 2000 has been used frequently to assess knee function in ACLR patients [8-10] and in patients with ACL deficiency [11-13].

The purpose of the KOS-ADLS is to identify symptoms and functional limitations during daily activities in patients with various knee pathologies. There are two versions of the KOS-ADLS: either 17 items or 14 items with the shorter version of the KOS-ADLS widely used in research. The 14 items version consists of two sections with 6 questions related to symptoms and 8 questions on functional limitations with activities of daily living. Patients rate items using descriptive responses. These responses are converted into a numerical ordinal scale for scoring with the scoring ranging from 5 for no symptoms or difficulty with activity to 0 for severe symptoms and difficulty with activities. The final KOSADLS score is represented as a percentage (\%) by using the equation presented below:

$$
\text { KOS-ADLS Score }=\left(\frac{\text { Sum of Items Answered }}{\text { Maximum Possible Score }}\right) \times 100
$$

Previous studies reported high test-retest reliability for KOS-ADLS between 0.94 and 0.98 [14-16] and this instrument demonstrates high internal consistency (Chronbach's alpha 0.89-0.98)[14]. Also, the KOSADLS shows good correlation with the Lysholm Knee Scoring Scale, global assessment of function and Western Ontario and McMaster Universities Osteoarthritis Index (WOMAC) subscales [15] indicating good construct validity. However, one limitation is that the items in the KOSADLS do not contain direct inputs from patients and thus this questionnaire may suffer from reduced content validity. However, a recent report showed that there are significant KOS-ADLS score differences between ACLR patients with normal knee function and abnormal knee function after both 6 months and 1 year post-ACLR [17]. Collectively, this previous literature indicates that KOS-ADLS questionnaire is an appropriate tool to assess knee function in post-ACLR patients.

The IKDC 2000 and the KOS-ADLS are frequently used instruments to evaluate patient-oriented subjective function in ACLR patients. Previous studies have confirmed that these tests exhibit high reliability and validity and can be useful to identify the current knee function of ACLR patients.

\section{OBJECTIVE FUNCTIONAL TESTS}

The quadriceps strength test most frequently used to evaluate ACLR patients level of function and readiness for return to activity is the maximum voluntary isometric contraction (MVIC) test [18]. One primary role of the quadriceps (knee extensor) muscle group is to eccentrically contract to control knee flexion and to slow down the body's center of mass during landing and cutting tasks [19]. Poor quadriceps muscle function has been linked to abnormal movement and asymmetrical loading patterns after ACLR [20-22]. Altered quadriceps muscle function was associated with sagittal plane knee biomechanics changes such as decreased knee flexion angle and decreased internal knee extension moment during walking and jogging [20,21]. In addition, Oberländer et al. [22] investigated landing mechanics during a single leg hop test in healthy individuals and those 6 months post ACLR and 12 months post ACLR. There was a strong correlation $\left(R^{2}=.78\right)$ between quadriceps MVIC and internal knee extension moment during a single leg hop test in individuals 12 months post ACLR. As described above, the quadriceps MVIC could be important factor to assess the muscle restoration, it represents a lack of overall function of the patient.

Recent studies have also investigated the relationship between the degree of quadriceps strength asymmetry (Quadriceps index [QI] = Injured limb/Uninjured limb $\times 100$ ), representing quadriceps strength difference between the injured and uninjured limbs, and biomechanical variables during a landing task $[23,24]$. ACLR individuals who presented lesser 
quadriceps strength symmetry $([\mathrm{QI}]<80)$ showed lesser peak knee flexion angle symmetry and peak internal knee extension moment symmetry during the single leg landing task compared to ACLR individuals who presented higher quadriceps strength symmetry $(\mathrm{QI} \geq 90)$ [23]. Similarly, Schmitt et al. [24] reported greater quadriceps strength asymmetry was associated with greater peak vertical ground reaction force and lesser peak internal knee extension moments during a drop vertical jump task. As the kinematic and kinetic differences reported in these studies have been identified as important biomechanical ACL injury risk factors $[23,24]$, these results support the idea that the restoration of quadriceps strength symmetry in ACLR patients' is likely important for decreasing second ACL injury risk.

In addition to quadriceps strength testing, single-leg hop tests are commonly used to assess ACLR patients' knee function. Single-leg hop testing was originally proposed by Noyes [25] and is composed of four different hop tests: single hop, triple hop, crossover hop, and 6-m timed hop [25]. Single hop, triple hop and crossover hop tests measure the total distance hopped, while the 6-meter hop is timed. These functional tests are reported to have high reliability to evaluate limb asymmetries defined as the between-limb difference in the distance hopped or time to hop 6 meters. In addition, they have been shown to be valid tests for evaluating ACLR patients' knee function with high test-retest reliability showing intraclass correlation coefficients ranging between 0.82-0.93 [26]. With respect for its utility for identifying functional limitations, a recent study reported that ACLR athletes who returned to pre-injury levels of sport activity at 2 years post-surgery showed higher single-leg hop and triple hop test limb symmetry indices compared to those who failed to return to pre-injury levels of activity [27]. Although the hop tests could be useful test to assess patient's lower extremity function, there is the possibility for patients to compensate movements which means patients completed this task with lack of quadriceps involvements.

\section{CUT-OFF CRITERIA FOR FUNCTIONAL MEASURES}

Several studies have used normative IKDC 2000 data for age and sex specific groups as a cut-off criteria to identify knee function [7,17]. The 15 th percentile from the normative data from uninjured female ages between $18-24$ is 83.9 of the IKDC 2000 score [7]. In many previous studies, greater than 85 of the IKDC 2000 has been frequently used as cutoff standard with this subjective measure [17,28-31]. Some studies used
KOS-ADLS as a test to decide ACLR patients' readiness of return to full activity $[17,32,33]$ and greater than $90 \%$ of the score was commonly used standard cut-off for this measure [17,33]. For objective functional tests such as quadriceps MVIC and single-leg hop tests, limb symmetry index (LSI) has been used to identify level of knee functional asymmetries $[17,32,34]$. LSI of $90 \%$, representing a $10 \%$ deficit in injured side limb function compared to uninjured side, has been widely used in previous studies as a cut-off standard [17,23,24,32,34-40].

\section{FUNCTIONAL TEST BATTERY}

A functional test battery (FTB), which incorporates several subjective and objective measures of knee function, has been proposed for use in determining individuals' readiness for return to activity by identifying limb asymmetries after ACL injury and reconstruction. The rationale underlying the use of multiple subjective and objective tests is that a FTB has been shown to more accurately discriminate neuromuscular function asymmetry between the injured and uninjured side of ACLR patients compared to single functional tests [41-43]. Gustavsson et al. [41] investigated the utilization of a three test FTB consisting of the vertical jump, the single-leg hop for distance, and the single-leg side hop. If a subject demonstrated a LSI $<90 \%$ on any of the three tests, the subject was classified as having failed the FTB. Among healthy groups (9 males and 6 females), 3 out of 15 (20\%) subjects were classified in fail group whereas 32 out of 35 (91\%) of ACLR patients (25 males and 10 females) were categorized as failing the FTB. Neeter et al. [42] utilized a different FTB that consisted of measuring knee extension, knee flexion, and leg press power (Watts). Ninety-five percent of patients 6 months post-ACLR were classified as abnormal ( $<90 \%$ LSI in at least one of three test). Logerstedt et al. [17] investigated whether the IKDC 2000 could discriminate between successful and non-successful performance on a FTB following ACLR. They reported that $78 \%$ of participants were classified as having normal knee function and 22\% were classified as having below normal knee function at 12 months after ACLR when using the IKDC 2000. However, among participants who were classified by the IKDC 2000 as having normal knee function, only $61.8 \%$ successfully passed a FTB. In addition, among participants who were classified as having below normal knee function by the IKDC 2000, 80.6\% failed a FTB.

Recently et al. [29] utilized a FTB that included both a patient-oriented subjective measure (IKDC 2000) and two objective functional measures (single-leg hop and triple hop for distance). This study then compared 
single leg squat biomechanics between ACLR patients that passed and failed the FTB. Subjects were categorized as passing the FTB if they scored greater than 85 on the IKDC 2000 and had LSIs $>90 \%$ for both the single and triple hop for distance. While only 7 and 9 individuals were classified the fail group when using only the single hop and triple hop for distance as their functional tests, respectively, 37 out of 66 (56\%) individuals were categorized in the fail group when all three measures were considered.

Collectively, these studies consistently showed that combination of functional measures; FTB, tend to better identify patients' functional limitation than using a single functional test. These findings may indicate that the use of FTB potentially is better to detect ACLR patients' knee function compare to the use of a single test. Therefore, it is possible that a FTB might be useful for identifying whether ACLR patients' can safely participate in full, unrestricted activity. The members of the European Board of Sports Rehabilitation (ESBR) proposed the recommended criteria for both strength and hop performance prior to return to full activity after ACLR based on previous published literatures and clinical experiences from ESBR members [44]. ESBR suggested 100\% LSI on knee extensor and knee flexor strength and 90\% on two maximum (Single hop/vertical hop) and one endurable hop (Triple hop or crossover hop) test for individuals who are participating in pivoting, contact, and competitive sports. For individuals who are participating in non-pivoting, non-contact, and recreational sports, they suggested 90\% LSI on knee extensor and knee flexor strength and 90\% LSI on one maximum or one endurable hop test as FTB.

\section{CONCLUSION}

There are various subjective and objective functional measures to assess ACLR patients' level of knee function. Those functional tests are relatively simple and easy to administer in a clinical setting. Also, many researchers have tried to find the optimal cut-off standard that indicates the safe level of knee function prior to return to full activity. A FTB is the concept of using the combination of several different subjective and objective functional tests. Since a FTB appears to demonstrate greater sensitivity for distinguishing ACLR individuals' knee function, recent studies have suggested utilizing FTBs to assess patients' readiness to safely participate in full activity. Further, despite the use of single functional tests, the risk for subsequent knee problems, most notably a second ACL injury, remains elevated in ACLR individuals. Therefore, it is necessary to investigate the usefulness of a FTB to identify individuals that are at greatest risk for second ACL injury.

\section{REFERENCES}

1. Paterno MV, Rauh MJ, Schmitt LC, Ford KR, Hewett TE. Incidence of contralateral and ipsilateral anterior cruciate ligament (ACL) injury after primary ACL reconstruction and return to sport. Clin J Sport Med. 2012;22(2):116-21.

2. Paterno MV, Rauh MJ, Schmitt LC, Ford KR, Hewett TE. Incidence of second ACL injuries 2 years after primary ACL reconstruction and return to sport. Am J Sports Med. 2014;42(7):1567-73.

3. Higgins LD, Taylor MK, Park D, Ghodadra N, Marchant M, et al. Reliability and validity of the International Knee Documentation Committee (IKDC) subjective knee form. Jt Bone Spine Rev Rhum. 2007;74(6):594-9.

4. Irrgang JJ, Anderson AF, Boland AL, Harner CD, Kurosaka M, et al. Development and validation of the international knee documentation committee subjective knee form. Am J Sports Med. 2001;29(5):60013.

5. Agel J, LaPrade RF. Assessment of differences between the modified cincinnati and international knee documentation committee patient outcome scores: a prospective study. Am J Sports Med. 2009;37(11):2151-7.

6. Haverkamp D, Sierevelt IN, Breugem SJM, Lohuis K, Blankevoort L, et al. Translation and validation of the dutch version of the international knee documentation committee subjective knee form. Am J Sports Med. 2006;34(10):1680-4.

7. Anderson AF, Irrgang JJ, Kocher MS, Mann BJ, Harrast JJ. The international knee documentation committee subjective knee evaluation form: normative data. Am J Sports Med. 2006;34(1):128-35.

8. Hambly K, Griva K. IKDC or KOOS which one captures symptoms and disabilities most important to patients who have undergone initial anterior cruciate ligament reconstruction?. Am J Sports Med. 2010; 38(7):1395-404.

9. Nyland J, Cottrell B, Harreld K, Caborn DNM. Self-reported outcomes after anterior cruciate ligament reconstruction: an internal health locus of control score comparison. Arthrosc J Arthrosc Relat Surg Off Publ Arthrosc Assoc N Am Int Arthrosc Assoc. 2006;22(11): 1225-32.

10. Spindler KP, Warren TA, Callison JC, Secic M, Fleisch SB, et al. Clinical outcome at a minimum of five years after reconstruction of the an- 
terior cruciate ligament. J Bone Joint Surg Am. 2005;87(8):1673-9.

11. Eastlack ME, Axe MJ, Snyder-Mackler L. Laxity, instability, and functional outcome after ACL injury: copers versus noncopers. Med Sci Sports Exerc. 1999;31(2):210-5.

12. Johnson DS, Smith RB. Outcome measurement in the ACL deficient knee-what's the score?. The Knee. 2001;8(1):51-7.

13. Park WH, Kim DK, Yoo JC, Lee YS, Hwang JH, et al. Correlation between dynamic postural stability and muscle strength, anterior instability, and knee scale in anterior cruciate ligament deficient knees. Arch Orthop Trauma Surg. 2010;130(8):1013-8.

14. Collins NJ, Misra D, Felson DT, Crossley KM, Roos EM. Measures of knee function: International Knee Documentation Committee (IKDC) subjective knee evaluation form, Knee Injury and Osteoarthritis Outcome Score (KOOS), Knee Injury and Osteoarthritis Outcome Score Physical Function Short Form (KOOS-PS), Knee Outcome Survey Activities of Daily Living Scale (KOS-ADL), lysholm knee scoring scale, Oxford Knee Score (OKS), Western Ontario and McMaster Universities Osteoarthritis Index (WOMAC), Activity Rating Scale (ARS), and Tegner Activity Score (TAS). Arthritis Care Res. 2011;63(S11):S208-28.

15. Irrgang JJ, Snyder-Mackler L, Wainner RS, Fu FH, Harner CD. Development of a patient-reported measure of function of the knee. J Bone Joint Surg Am. 1998;80(8):1132-45.

16. Marx RG, Jones EC, Allen AA, Altchek DW, O’Brien SJ, et al. Reliability, validity, and responsiveness of four knee outcome scales for athletic patients. J Bone Joint Surg Am. 2001;83-A(10):1459-69.

17. Logerstedt D, Di Stasi S, Grindem H, Lynch A, Eitzen I, et al. Self-reported knee function can identify athletes who fail return-to-activity criteria up to 1 year after anterior cruciate ligament reconstruction: a delaware-oslo ACL cohort study. J Orthop Sports Phys Ther. 2014 Dec;44(12):914-23.

18. Barber-Westin SD, Noyes FR. Factors used to determine return to unrestricted sports activities after anterior cruciate ligament reconstruction. Arthrosc J Arthrosc Relat Surg Off Publ Arthrosc Assoc N Am Int Arthrosc Assoc. 2011;27(12):1697-705.

19. Winter DA. Biomechanics and motor control of human movement fourth edition. John Wiley \& Sons, Inc 2005.

20. Bush-Joseph CA, Hurwitz DE, Patel RR, Bahrani Y, Garretson R, et al. Dynamic function after anterior cruciate ligament reconstruction with autologous patellar tendon. Am J Sports Med. 2001;29(1):36-41.

21. Lewek M, Rudolph K, Axe M, Snyder-Mackler L. The effect of insuffi- cient quadriceps strength on gait after anterior cruciate ligament reconstruction. Clin Biomech Bristol Avon. 2002;17(1):56-63.

22. Oberländer KD, Brüggemann GP, Hoeher J, Karamanidis K. Altered landing mechanics in ACL-reconstructed patients. Med Sci Sports Exerc. 2013;45(3):506-13.

23. Palmieri-Smith RM, Lepley LK. Quadriceps strength asymmetry after anterior cruciate ligament reconstruction alters knee joint biomechanics and functional performance at time of return to activity. Am J Sports Med. 2015;43(7):1662-9.

24. Schmitt LC, Paterno MV, Ford KR, Myer GD, Hewett TE. Strength asymmetry and landing mechanics at return to sport after ACL reconstruction. Med Sci Sports Exerc. 2014;47(7):1426-34.

25. Noyes FR, Barber SD, Mangine RE. Abnormal lower limb symmetry determined by function hop tests after anterior cruciate ligament rupture. Am J Sports Med. 1991;19(5):513-8.

26. Reid A, Birmingham TB, Stratford PW, Alcock GK, Giffin JR. Hop testing provides a reliable and valid outcome measure during rehabilitation after anterior cruciate ligament reconstruction. Phys Ther. 2007; 87(3):337-49.

27. Ardern CL, Taylor NF, Feller JA, Whitehead TS, Webster KE. Sports participation 2 years after anterior cruciate ligament reconstruction in athletes who had not returned to sport at 1 year: a prospective followup of physical function and psychological factors in 122 athletes. Am J Sports Med. 2015;43(4):848-56.

28. Ardern CL, Webster KE, Taylor NF, Feller JA. Return to the preinjury level of competitive sport after anterior cruciate ligament reconstruction surgery: two-thirds of patients have not returned by 12 months after surgery. Am J Sports Med. 2011;39(3):538-43.

29. Bell D, Kulow S, Pennuto A, Stiffler M. Squatting mechanics between ACL-Reconstructed patients who pass and fail return-to-sport criteria. J Athl Train. 2014;49(3 Suppl):S237.

30. Kulow SM, Pennuto AP, Gire CC, Stiffler MR, Bell DR. Jump landing mechanics in those who pass and fail return to sport criteria. J Athl Train. 2014;49(3 Suppl):S122.

31. Logerstedt D, Grindem H, Lynch A, Eitzen I, Engebretsen L, et al. Single-legged hop tests as predictors of self-reported knee function after anterior cruciate ligament reconstruction: the Delaware-Oslo ACL cohort study. Am J Sports Med. 2012;40(10):2348-56.

32. Fitzgerald GK, Axe MJ, Snyder-Mackler L. A decision-making scheme for returning patients to high-level activity with nonoperative treatment after anterior cruciate ligament rupture. Knee Surg Sports Trau- 
matol Arthrosc Off J ESSKA. 2000;8(2):76-82.

33. Hartigan EH, Axe MJ, Snyder-Mackler L. Time line for noncopers to pass return-to-sports criteria after anterior cruciate ligament reconstruction. J Orthop Sports Phys Ther. 2010;40(3):141-54.

34. Xergia SA, Pappas E, Zampeli F, Georgiou S, Georgoulis AD. Asymmetries in functional hop tests, lower extremity kinematics, and isokinetic strength persist 6 to 9 months following anterior cruciate ligament reconstruction. J Orthop Sports Phys Ther. 2013;43(3):154-62.

35. Engelen-van Melick N, van Cingel REH, Tijssen MPW, Nijhuis-van der Sanden MWG. Assessment of functional performance after anterior cruciate ligament reconstruction: a systematic review of measurement procedures. Knee Surg Sports Traumatol Arthrosc Off J ESSKA. 2013;21(4):869-79.

36. Greenberg EM, Greenberg ET, Ganley TJ, Lawrence JTR. Strength and functional performance recovery after anterior cruciate ligament reconstruction in preadolescent athletes. Sports Health. 2014;6(4):30912.

37. Hildebrandt C, Müller L, Zisch B, Huber R, Fink C, et al. Functional assessments for decision-making regarding return to sports following ACL reconstruction. Part I: development of a new test battery. Knee Surg Sports Traumatol Arthrosc Off J ESSKA. 2015;23(5):1273-81.

38. Lepley LK, Wojtys EM, Palmieri-Smith RM. Combination of eccentric exercise and neuromuscular electrical stimulation to improve biomechanical limb symmetry after anterior cruciate ligament reconstruction. Clin Biomech Bristol Avon. 2015;30(7):738-47.
39. Myer GD, Martin L, Ford KR, Paterno MV, Schmitt LC, et al. No association of time from surgery with functional deficits in athletes after anterior cruciate ligament reconstruction: evidence for objective return-to-sport criteria. Am J Sports Med. 2012;40(10):2256-63.

40. Rohman E, Steubs JT, Tompkins M. Changes in involved and uninvolved limb function during rehabilitation after anterior cruciate ligament reconstruction: implications for limb symmetry index measures. Am J Sports Med. 2015;43(6):1391-8.

41. Gustavsson A, Neeter C, Thomeé P, Silbernagel KG, Augustsson J, et al. A test battery for evaluating hop performance in patients with an ACL injury and patients who have undergone ACL reconstruction. Knee Surg Sports Traumatol Arthrosc Off J ESSKA. 2006;14(8):77888.

42. Neeter C, Gustavsson A, Thomeé P, Augustsson J, Thomeé R, et al. Development of a strength test battery for evaluating leg muscle power after anterior cruciate ligament injury and reconstruction. Knee Surg Sports Traumatol Arthrosc Off J ESSKA. 2006;14(6):571-80.

43. Thomeé R, Neeter C, Gustavsson A, Thomeé P, Augustsson J, et al Variability in leg muscle power and hop performance after anterior cruciate ligament reconstruction. Knee Surg Sports Traumatol Arthrosc Off J ESSKA. 2012;20(6):1143-51.

44. Thomeé R, Kaplan Y, Kvist J, Myklebust G, Risberg MA, et al. Muscle strength and hop performance criteria prior to return to sports after ACL reconstruction. Knee Surg Sports Traumatol Arthrosc Off J ESSKA. 2011;19(11):1798-805. 